\title{
Review Article \\ On How QCD Gauge Invariance Gets Realized in the Context of Effective Locality
}

\author{
H. M. Fried, ${ }^{1}$ T. Grandou $(\mathbb{D})^{2}$ and R. Hofmann ${ }^{3}$ \\ ${ }^{1}$ Physics Department, Brown University, Providence, RI 02912, USA \\ ${ }^{2}$ Institut de Physique de Nice, UMR CNRS 7010, Université de Nice-Sophia Antipolis, 1361 route des Lucioles, 06560 Valbonne, France \\ ${ }^{3}$ Institut für Theoretische Physik, Universität Heidelberg, Philosophenweg 16, 69120 Heidelberg, Germany
}

Correspondence should be addressed to T. Grandou; thierry.grandou@inphyni.cnrs.fr

Received 23 August 2017; Accepted 29 March 2018; Published 22 May 2018

Academic Editor: Enrico Lunghi

Copyright (C) 2018 H. M. Fried et al. This is an open access article distributed under the Creative Commons Attribution License, which permits unrestricted use, distribution, and reproduction in any medium, provided the original work is properly cited. The publication of this article was funded by $\mathrm{SCOAP}^{3}$.

The fermionic Green's functions of QCD exhibit an unexpected property of effective locality, which appears to be exact, involving no approximation. This property is nonperturbative, resulting from a full integration of the elementary gluonic degrees of freedom of QCD. Recalling, correcting, and extending the derivations of effective locality, focus is put on the way nonabelian gauge invariance gets realized in the fermionic nonperturbative regime of QCD.

\section{Introduction}

In some recent articles [1-5], a property, which bears on the nonperturbative fermionic Green's functions of QCD, has been put forth under the name of effective locality (EL). This property can be summarized as follows.

For any fermionic $2 n$-point Green's functions and related amplitudes, the full gauge-fixed sum of cubic and quartic gluonic interactions, with fermionic loops included, results in a local contact-type interaction. This local interaction is mediated by a tensorial field which is antisymmetric both in Lorentz and color indices. Moreover, the resulting sum appears to be fully gauge-fixing independent, that is, gauge-invariant.

This is an unexpected result because integrations of elementary degrees of freedom ordinarily result in highly nonlocal structures. The "effective locality" denomination, which sounds like an oxymoron, accounts for this unusual circumstance. It is worth pointing out that in the pure euclidean Yang Mills case and up to the first nontrivial orders of a semiclassical expansion, effective locality was observed as a welcome property in an attempt to construct a formulation dual to the original Yang Mills theory [6-8].

Now, apart from a supersymmetric extension, QCD is not known for possessing any dual formulation and the full
EL functional expressions certainly attest to this difficulty. It remains that, like in the pure Yang Mills situation of $[6,7]$, the EL property may allow one to learn something about the nonperturbative regime of QCD and this from first principles.

In the next section a comparison of the QED and QCD Green's functions generating functional is given, while Section 3 displays the property of effective locality in the simpler situation of eikonal and quenching approximations where it was first noticed and then in full generality. Eventually, Section 4 presents some concluding remarks.

\section{Contrasting Generating Functionals}

In QED it has been known for quite a long time that manifest covariance and manifest gauge invariance are competing aspects of a generating functional construction. For short, starting from a photonic Lagrangian density of

$$
\mathscr{L}_{0}=-\frac{1}{4} f^{\mu \nu} f_{\mu \nu}=-\frac{1}{4}\left(\partial^{\mu} A^{\nu}-\partial^{\nu} A^{\mu}\right)\left(\partial_{\mu} A_{\nu}-\partial_{\nu} A_{\mu}\right),
$$

and corresponding action (at $\lambda=1$ ),

$$
\int \mathrm{d}^{4} x \mathscr{L}_{0}=-\frac{1}{2} \int A^{\mu}\left(-\partial^{2}\right) A_{\mu}+\frac{\lambda}{2} \int\left(\partial^{\mu} A_{\mu}\right)^{2},
$$


one may use the "inconvenient" (in Fermi's words [1]) term of $(\partial \cdot A)^{2}$ to generate any relativistic gauge (manifest covariance), treating, for example, $(\lambda / 2) \int\left(\partial^{\mu} A_{\mu}\right)^{2}$ as a kind of "interaction." Gauge invariance is then guaranteed by enforcing charge conservation: clearly not a manifest way.

The construction can be summarized through the following steps. Starting from the free-field case $(\lambda=e=0)$, one has, with $D_{F \mu \nu}^{(0)}=g_{\mu \nu} D_{F},\left(-\partial^{2}\right) D_{F}=1$, the free-field generating functional

$$
Z_{0}^{(0)}\{j\}=\exp \left\{\frac{i}{2} \int j \cdot D_{\mathrm{F}}^{(0)} \cdot j\right\}
$$

Then one can proceed in Schwinger's way with the "gaugeinteraction term":

$$
\begin{aligned}
Z_{0}^{(\zeta)}\{j\} & =\left.e^{i(\lambda / 2) \int\left(\partial^{\mu} A_{\mu}\right)^{2}}\right|_{A \rightarrow(1 / i)(\delta / \delta j)} \cdot e^{(i / 2) \int j \cdot D_{\mathrm{F}}^{(0)} \cdot j} \\
& =e^{-(i / 2) \operatorname{Tr} \ln \left[1-\lambda\left(\partial \otimes \partial / \partial^{2}\right)\right]} e^{(i / 2) \int j \cdot D_{\mathrm{F}}^{(\zeta)} \cdot j}
\end{aligned}
$$

with

$$
D_{\mathrm{F} \mu \nu}^{(\zeta)}=\left[g_{\mu \nu}-\frac{\zeta \partial_{\mu} \partial_{\nu}}{\partial^{2}}\right] D_{\mathrm{F}}, \quad \zeta=\frac{\lambda}{1-\lambda} .
$$

The full Green's functions generating functional now reads as $(\lambda \neq 0, e \neq 0)$

$$
\begin{aligned}
& Z_{\mathrm{QED}}^{(\zeta)}[j, \eta, \bar{\eta}] \\
& =\left.\mathcal{N} e^{i \int \bar{\eta} \cdot G_{\mathrm{F}}[A] \cdot \eta+L[A]+(i / 2) \lambda \int\left(\partial^{\mu} A_{\mu}\right)^{2}}\right|_{A \rightarrow(1 / i)(\delta / \delta j)} \\
& \quad \cdot e^{(i / 2) \int j \cdot D_{\mathrm{F}}^{(0)} \cdot j}
\end{aligned}
$$

with (the phase factor appearing in the second line of (4) is absorbed into the normalization constant $\mathcal{N}$ )

$$
\begin{aligned}
G_{F}[A] & =[\gamma \cdot(\partial-i e A)-m]^{-1}, \\
L[A] & =\operatorname{Tr} \ln \left[1-i e \gamma \cdot A S_{\mathrm{F}}\right], \quad S_{\mathrm{F}}=G_{\mathrm{F}}[0] .
\end{aligned}
$$

This is the standard Schwinger solution for the QED generating functional. For any polynomial and/or exponential functional $\mathscr{F}[A]$, the following differential functional identity holds:

$$
\left.\mathscr{F}\left[\frac{1}{i} \frac{\delta}{\delta j}\right] \cdot e^{(i / 2) \int j \cdot D_{\mathrm{F}}^{(\zeta)} \cdot j} \equiv e^{(i / 2) \int j \cdot D_{\mathrm{F}}^{(\zeta)} \cdot j} \cdot e^{\mathfrak{D}_{A}} \cdot \mathscr{F}[A]\right|_{A=\int D_{\mathrm{F}}^{(\zeta)} \cdot j}
$$

with the so-called linkage operator

$$
\mathfrak{D}_{A}^{(\zeta)}=-\frac{i}{2} \int \mathrm{d}^{4} x \int \mathrm{d}^{4} y \frac{\delta}{\delta A(x)} \cdot D_{\mathrm{F}}^{(\zeta)}(x-y) \cdot \frac{\delta}{\delta A(y)} .
$$

This identity can be used to write eventually

$$
\begin{aligned}
Z_{\mathrm{QED}}^{(\zeta)}[j, \eta, \bar{\eta}]= & \mathcal{N} e^{(i / 2) \int j \cdot D_{\mathrm{F}}^{(\zeta)} \cdot j} \\
& \left.\cdot e^{\mathfrak{D}_{A}^{(\zeta)}} \cdot e^{i \int \bar{\eta} \cdot G_{\mathrm{F}}[A] \cdot \eta+L[A]}\right|_{A=\int D_{\mathrm{F}}^{(\zeta)} \cdot j} .
\end{aligned}
$$

Of course, this is the QED generating functional that would result from linear and covariant gauge fixing of $(-1 / 2(1-$ $\lambda))(\partial \cdot A)^{2}$. However, $\lambda=1$ cannot be chosen as $D_{\mathrm{F} \mu \nu}^{(\zeta)}$ would not be defined. It is at $\lambda=1$ though that manifest gauge invariance is maintained. As a result, Green's functions cannot display gauge invariance. As shown in (10), the bare photon propagator remains explicitly gauge-dependent, and through Ward-Takahashi identities, its radiative corrections are checked to be gauge-invariant. For the whole set of physical processes described by QED, the LSZ reduction formula and equivalence theorem [9] allow one to pass from nongauge-invariant Green's functions to gauge-invariant $S$ matrix elements. Recovering gauge invariance is therefore a circuitous procedure and it seems that the nonabelian structure of QCD offers a different possibility.

In the case of QCD, the same canonical steps can be taken. To sum up and within concise notations (color degrees being understood)

$$
\begin{aligned}
Z_{\mathrm{QCD}}^{(?)}[j, \bar{\eta}, \eta] & =\mathcal{N} e^{i \int \mathscr{L}^{\prime}{ }_{\mathrm{QCD}}[(1 / i)(\delta / \delta j)]} e^{(i / 2) \int j \cdot D_{\mathrm{F}}^{(?)} \cdot j}, \\
\mathscr{L}_{\mathrm{QCD}}^{\prime}[A] & =\bar{\eta} G_{\mathrm{F}}[A] \eta+L[A]+\mathscr{L}^{\prime}[A],
\end{aligned}
$$

where the following propagator, of course, does not exist:

$$
\left(D_{\mathrm{F}}^{(?)^{-1}}\right)_{\mu \nu}^{a b}=-i \delta^{a b}\left[g_{\mu \nu} \partial^{2}-\partial_{\mu} \partial_{\nu}\right]
$$

However, in QCD, unlike QED, one has

$$
\begin{aligned}
\int \mathscr{L}^{\prime}[A]= & -\frac{1}{4} \int F^{2}-\frac{1}{2} \int A_{a}^{\mu}\left(\partial^{2}\right) A_{\mu}^{a} \\
& -\frac{1}{2} \int\left(\partial^{\mu} A_{\mu}^{a}\right)^{2},
\end{aligned}
$$

that is, omitting fermionic variables momentarily,

$$
\begin{aligned}
Z^{(?)}[j]= & \left.\mathscr{N} e^{-(i / 4) \int F^{2}+(i / 2) \int A_{\mu}^{a}\left(D_{\mathrm{F}}^{(?)}\right)^{-1} A_{a}^{\mu}}\right|_{A \rightarrow(1 / i)(\delta / \delta j)} \\
& \cdot e^{(i / 2) \int j \cdot D_{\mathrm{F}}^{(?)} \cdot j} .
\end{aligned}
$$

Now, simply adding and subtracting a linear covariant gauge-fixing term of $(1 / 2 \zeta)(\partial \cdot A)^{2}$, it is elementary to proceed and pass from an undefined $D_{\mathrm{F}}^{(?)}$ to the well-defined and covariant propagator $D_{\mathrm{F}}^{(\zeta)}$ :

$$
\left(D_{\mathrm{F}}^{(\zeta)^{-1}}\right)_{\mu \nu}^{a b}=-i \delta^{a b}\left[g_{\mu \nu} \partial^{2}+\left(\frac{1}{\zeta}-1\right) \partial_{\mu} \partial_{\nu}\right]
$$

and to a Green's function QCD generating functional of

$$
\begin{aligned}
& Z_{\mathrm{QCD}}[j, \bar{\eta}, \eta]=\mathcal{N} e^{(i / 2) \int j \cdot D_{\mathrm{F}}^{(\zeta)} \cdot j} e^{-(i / 2) \int(\delta / \delta A) \cdot D_{\mathrm{F}}^{(\zeta)} \cdot(\delta / \delta A)} \\
& \times e^{-(i / 4) \int F^{2}+(i / 2) \int A \cdot\left(D_{\mathrm{F}}^{(\zeta)}\right)^{-1} \cdot A} \\
& \left.\cdot e^{i \int \bar{\eta} \cdot G_{\mathrm{F}}[A] \cdot \eta+L[A]}\right|_{A=\int D_{\mathrm{F}}^{(\zeta)} \cdot j}
\end{aligned}
$$


where fermionic dependence has been restored and the differential functional identity (8) used again.

However, likewise, adding and subtracting a gauge fixing of $(1 / 2 \xi)(n \cdot A)^{2}$, with $n$ being a lightlike vector, $n^{2}=0$, one gets (in the limit $\xi \rightarrow 0$, [9])

$$
\left(D_{\mathrm{F}}^{(n)}\right)_{\mu \nu}^{a b}(K)=-i \delta^{a b}\left(\frac{g_{\mu \nu}}{K^{2}+i \varepsilon}-\frac{K_{\mu} n_{\nu}+K_{v} n_{\mu}}{\left(K^{2}+i \varepsilon\right)(K \cdot n)}\right)
$$

and a Green's function QCD generating functional of

$$
\begin{aligned}
& Z_{\mathrm{QCD}}[j, \bar{\eta}, \eta]=\mathscr{N} e^{(i / 2) \int j \cdot D_{\mathrm{F}}^{(n)} \cdot j} e^{-(i / 2) \int(\delta / \delta A) \cdot D_{\mathrm{F}}^{(n)} \cdot(\delta / \delta A)} \\
& \times e^{-(i / 4) \int F^{2}+(i / 2) \int A \cdot\left(D_{\mathrm{F}}^{(n)}\right)^{-1} \cdot A} \\
& \left.\cdot e^{i \int \bar{\eta} \cdot G_{\mathrm{F}}[A] \cdot \eta+L[A]}\right|_{A=\int D_{\mathrm{F}}^{(n)} \cdot j} \cdot
\end{aligned}
$$

As many identical forms as desired can be obtained for $Z_{\mathrm{QCD}}[j, \bar{\eta}, \eta]$, differing only in the intermediate gluonic field function, $D_{F}^{(n)}, D_{F}^{(\zeta)}$, and so forth.

In conclusion, the following points can be proposed.

(i) In QED (where $\lambda=1$ cannot be chosen), these standard functional operations amount to a quantization in a given covariant gauge. Manifest Lorentz covariance is maintained while manifest gauge invariance is not. Gauge invariance is the matter of indirect recovering.

(ii) In QCD, surprisingly and explicitly due to the nonabelian structure, manifest Lorentz covariance and manifest gauge invariance can be preserved at a time.

(iii) Since no gauge fixing is really achieved, none of the several forms of the QCD generating functional, with $D_{\mathrm{F}}^{(n)}$, $D_{\mathrm{F}}^{(\zeta)}$, or any other free-field function, do indeed correspond to the usual QCD generating functional in the associated gauge.

(iv) There is but one possibility. Gauge field functions (i.e., propagators) must show up as necessary intermediates and not affect the fermionic momenta of the QCD generating functionals (16) and (18).

(v) Now, this is guaranteed by the property of effective locality.

\section{Effective Locality}

The property of effective locality will be first displayed in a simplified situation corresponding to the use of eikonal and quenching approximations.

3.1. Effective Locality in a Small Example: Eikonal and Quenching Approximations. With the help of the representation [10],

$$
e^{-(i / 4) \int F^{2}}=\mathcal{N}^{\prime} \int \mathrm{d}[\chi] e^{(i / 4) \int\left(\chi_{\mu \nu}^{a}\right)^{2}+(i / 2) \int \chi_{a}^{\mu \nu} F_{\mu \nu}^{a}}
$$

any $D_{F}$-dependent form of $Z_{\mathrm{QCD}}[j, \bar{\eta}, \eta]$ can be used; for example,

$$
\begin{aligned}
& \mathcal{N} e^{(i / 2) \int j \cdot D_{\mathrm{F}}^{(0)} \cdot j} \int \mathrm{d}[\chi] \\
& \cdot e^{(i / 4) \int \chi^{2}} e^{\mathfrak{D}_{A}^{(0)}} e^{-(i / 2) \int \chi \cdot F+(i / 2) \int A \cdot\left(-\partial^{2}\right) \cdot A} \\
& \left.\cdot e^{i \int \bar{\eta} \cdot G_{\mathrm{F}}[A] \cdot \eta+L[A]}\right|_{A=\int D_{\mathrm{F}}^{(0)} j} \cdot
\end{aligned}
$$

However, representation (19) breaks the manifest gauge invariance. This can be remedied either by gauging the $\chi$ field $[6,7]$ or by means of an exact integration of the $\chi$ field dependence. In the latter instance, the powerful Random Matrix theory allows one to do it in the "small case" at least [4]. So long as gauge invariance is concerned, however, none of the above possibilities is necessary as effective locality brings about a remarkable simplification.

Let there be a Fradkin's representation of the functional $G_{F}[A][11]$ :

$$
\begin{aligned}
& \left\langle p\left|G_{F}[A]\right| y\right\rangle=e^{-i p \cdot y} i \int_{0}^{\infty} \mathrm{d} s e^{-i s m^{2}} e^{-(1 / 2) \operatorname{Tr} \ln (2 h)} \\
& \times \int \mathrm{d}[u]\{m-i \gamma \cdot[p-g A(y-u(s))]\} \\
& \cdot e^{(i / 4) \int_{0}^{s} \mathrm{~d} s^{\prime}\left[u^{\prime}\left(s^{\prime}\right)\right]^{2}} e^{i p \cdot u(s)} \\
& \quad \times\left(e^{g \int_{0}^{s} \mathrm{~d} s^{\prime} \sigma \cdot \mathbf{F}\left(y-u\left(s^{\prime}\right)\right)} e^{-i g \int_{0}^{s} \mathrm{~d} s^{\prime} u^{\prime}\left(s^{\prime}\right) \cdot \mathbf{A}\left(y-u\left(s^{\prime}\right)\right)}\right)_{+}
\end{aligned}
$$

where $u(s)$ is the 4 -vector Fradkin's field variable, the subscript + stands for $s$-Schwinger proper-time ordering, and $h\left(s_{1}, s_{2}\right)=\int_{0}^{s} \mathrm{~d} s^{\prime} \Theta\left(s_{1}-s^{\prime}\right) \Theta\left(s_{2}-s^{\prime}\right)$. This representation is exact but fairly involved. In eikonal approximation $(u(s)=$ $s p$ ) the ordered exponential reduces to

$$
\begin{aligned}
\left(e^{i g p^{\mu} \int_{-\infty}^{+\infty} \mathrm{d} s A_{\mu}^{a}(y-s p) T^{a}}\right)_{+} \\
=\mathcal{N} \int \mathrm{d}[\alpha] \int \mathrm{d}[\Omega] \\
\quad \times e^{-i \int_{-\infty}^{+\infty} \mathrm{d} s \Omega^{a}(s)\left[\alpha^{a}(s)-g p^{\mu} A_{\mu}^{a}(y-s p)\right]} \\
\quad \times\left(e^{i \int_{-\infty}^{+\infty} \mathrm{d} s \alpha^{a}(s) T^{a}}\right)_{+}
\end{aligned}
$$

In (22), the $\alpha, \Omega$ are auxiliary variables introduced to take $A_{\mu}^{a}$-field dependence out of the ordered exponential; they are to be integrated out exactly so that dealing with the original time-ordered expressions is guaranteed. Again, this is doable at least in the strong coupling limit of $g \gg 1$ of a quenching approximation $(L[A]=0)$. 
A 4-pt Green's function illustration can be given. One has basically the expression

$$
\begin{gathered}
\prod_{i=1}^{2} \int \mathrm{d} s_{i} \int \mathrm{d}\left[u_{i}\left(s_{i}\right)\right] \int \mathrm{d}\left[\alpha_{i}\left(s_{i}\right)\right] \int \mathrm{d}\left[\Omega_{i}\left(s_{i}\right)\right](\cdot) \\
\int \mathrm{d}[\chi] e^{(i / 4) \int \chi^{2}} e^{\mathfrak{D}_{A}^{(0)}} e^{+(i / 2) \int A_{\mu}^{a} K_{a b}^{\mu \nu} A_{\nu}^{b}} e^{\left.i \int Q_{\mu}^{a} A_{a}^{\mu}\right|_{A \rightarrow 0},} \\
K_{\mu \nu}^{a b}=g f^{a b c} \chi_{\mu \nu}^{c}+\left(D_{\mathrm{F}}^{(0)^{-1}}\right)_{\mu \nu}^{a b}, \\
Q_{\mu}^{a}=-\partial^{\nu} \chi_{\mu \nu}^{a}+g\left[R_{1, \mu}^{a}+R_{2, \mu}^{a}\right], \\
R_{i, \mu}^{a}(z)=p_{i, \mu} \int \mathrm{d} s_{i} \Omega_{i}^{a}\left(s_{i}\right) \delta^{4}\left(z-y_{i}+s_{i} p_{i}\right),
\end{gathered}
$$

where the dots in the first line of (23) account for the integrand

$$
\begin{gathered}
e^{-i s_{i} m^{2}} e^{-(1 / 2) \operatorname{Tr} \ln (2 h)} e^{(i / 4) \int_{0}^{s_{i}} \mathrm{~d} s_{i}^{\prime}\left[u_{i}^{\prime}\left(s_{i}^{\prime}\right)\right]^{2}} \delta^{(4)}\left(x_{i}-y_{i}\right. \\
\left.+u_{i}\left(s_{i}\right)\right) e^{-i \int \mathrm{d} s_{i}^{\prime} \Omega^{a}\left(s_{i}^{\prime}\right) \alpha^{a}\left(s_{i}^{\prime}\right)}\left(e^{i \int_{-\infty}^{+\infty} \mathrm{d} s_{i} \alpha_{i}^{a}\left(s_{i}\right) \lambda^{a}}\right)_{+} .
\end{gathered}
$$

Now,

$$
\begin{gathered}
\left.e^{-(i / 2) \int(\delta / \delta A) \cdot D_{\mathrm{F}}^{(0)} \cdot(\delta / \delta A)} \cdot e^{+(i / 2) \int A \cdot K \cdot A+i \int A \cdot Q}\right|_{A \rightarrow 0} \\
=e^{-(1 / 2) \operatorname{Tr} \ln \left(1-D_{\mathrm{F}}^{(0)} \cdot K\right)} \cdot e^{(i / 2) \int Q \cdot\left[D_{\mathrm{F}}^{(0)} \cdot\left(1-K \cdot D_{\mathrm{F}}^{(0)}\right)^{-1}\right] \cdot Q},
\end{gathered}
$$

with

$$
\begin{aligned}
D_{\mathrm{F}}^{(0)} & \cdot\left(1-K \cdot D_{\mathrm{F}}^{(0)}\right)^{-1} \\
& =D_{\mathrm{F}}^{(0)} \cdot\left(1-\left[g f \cdot \chi+D_{\mathrm{F}}^{(0)^{-1}}\right] \cdot D_{\mathrm{F}}^{(0)}\right)^{-1} \\
& =-(g f \cdot \chi)^{-1},
\end{aligned}
$$

and the locality

$$
\left\langle x\left|(g f \cdot \chi)^{-1}\right| y\right\rangle=(g f \cdot \chi)^{-1}(x) \delta^{(4)}(x-y) .
$$

The 4-pt fermionic Green's function finally reads as

$$
\mathscr{N} \prod_{i=1}^{2} \int \mathrm{d} s_{i} \int \mathrm{d}\left[u_{i}\left(s_{i}\right)\right] \int \mathrm{d}\left[\alpha_{i}\left(s_{i}\right)\right] \int \mathrm{d}\left[\Omega_{i}\left(s_{i}\right)\right](\cdot) \int \mathrm{d}[\chi] e^{(i / 4) \int \chi^{2}} \cdot \frac{1}{\sqrt{\operatorname{det}(f \cdot \chi)}} \cdot e^{-(i / 2) \int \mathrm{d}^{4} z \mathrm{Q}(z) \cdot(g f \cdot \chi(z))^{-1} \cdot \mathrm{Q}(z)}
$$

with $Q_{\mu}^{a}(z)$ being the "currents" given in (24).

(i) This result is obviously gauge-independent, that is, gauge-invariant. It cannot show up in the abelian QED case as long hoped (R. P. Feynman, quoted in [12]). It generalizes to the case of $2 n$-point fermionic Green's functions $[13,14]$. When truncated to the YM case, the structure found in $[6,7]$ is basically reproduced. Eventually, this result lends itself to a ( $\mathrm{n}$ analytically continued) Random Matrix exact calculation which takes care of the full integration of the $\chi$ field dependence and displays the full algebraic dependence of fermionic Green's functions on the $S U_{c}(3)$-Lie algebra invariants $[13,14]$.

3.2. Effective Locality in the General Case. It is possible to relax any of the approximations used in the "small case" and end up with the property of effective locality. Again this can be illustrated in the simpler case of a fermionic 4-point Green's function:

$$
\begin{aligned}
& M\left(x_{1}, y_{1} ; x_{2}, y_{2}\right)=\frac{\delta}{\delta \bar{\eta}\left(y_{1}\right)} \cdot \frac{\delta}{\delta \eta\left(x_{1}\right)} \cdot \frac{\delta}{\delta \bar{\eta}\left(y_{2}\right)} \\
& \left.\quad \cdot \frac{\delta}{\delta \eta\left(x_{2}\right)} \cdot \mathscr{Z}\{j, \bar{\eta}, \eta\}\right|_{\eta=\bar{\eta}=0 ; j=0}=\mathcal{N} \int \mathrm{d}[\chi] \\
& \cdot e^{(i / 4) \int \chi^{2}} e^{\mathfrak{D}_{A}^{(\zeta)}} e^{+(i / 2) \int \chi \cdot \mathbf{F}+(i / 2) \int A \cdot\left(\mathbf{D}_{F}^{(\zeta)}\right)^{-1} \cdot A} G_{\mathrm{F}}\left(x_{1}, y_{1} \mid\right. \\
& \text { gA) }\left.G_{\mathrm{F}}\left(x_{2}, y_{2} \mid g A\right) e^{L[A]}\right|_{A=0} .
\end{aligned}
$$

Gathering Gaussian $A_{\mu}^{a}$ dependence of $G_{F}(1 \mid A)$ and $G_{F}(2 \mid$ A),

$$
\begin{aligned}
& \exp \left[\frac{i}{2} \int \mathrm{d}^{4} z A_{a}^{\mu}(z) K_{\mu \nu}^{a b}(z) A_{b}^{\nu}(z)\right. \\
& \left.\quad+i \int \mathrm{d}^{4} z Q_{a}^{\mu}(z) A_{\mu}^{a}(z)\right]
\end{aligned}
$$

requires 2 extra auxiliary variables; with

$$
\Phi_{i, \mu \nu}^{a}\left(u_{i} ; z\right) \equiv \int_{0}^{s_{i}} \mathrm{~d} s_{i}^{\prime} \delta^{(4)}\left(z-y_{i}+u_{i}\left(s_{i}^{\prime}\right)\right) \Phi_{i, \mu \nu}^{a}\left(s_{i}^{\prime}\right)
$$

a new piece of "interaction kernel" reads

$$
\begin{aligned}
& K_{\operatorname{spin}_{\mu \nu}}^{a b}(z) \\
& =2 g^{2} \int_{0}^{s_{1}} \mathrm{~d} s_{1}^{\prime} \delta^{(4)}\left(z-y_{1}+u_{1}\left(s_{1}^{\prime}\right)\right) f^{a b c} \Phi_{\mu \nu, 1}^{c}\left(s_{1}^{\prime}\right) \\
& \quad+2 g^{2} \int_{0}^{s_{2}} \mathrm{~d} s_{2}^{\prime} \delta^{(4)}\left(z-y_{2}+u_{2}\left(s_{2}^{\prime}\right)\right) f^{a b c} \Phi_{\mu \nu, 2}^{c}\left(s_{2}^{\prime}\right)
\end{aligned}
$$

while "currents" $Q_{\mu \nu}^{a}(z)$ get completed into

$$
\begin{aligned}
& Q_{\mu}^{a}(z) \\
& =-2 g \partial^{\nu} \Phi_{\mathrm{I}, \nu \mu}^{a}(z)
\end{aligned}
$$




$$
\begin{aligned}
& -g \int_{0}^{s_{1}} \mathrm{~d} s_{1}^{\prime} \delta^{(4)}\left(z-y_{1}+u_{1}\left(s_{1}^{\prime}\right)\right) u_{1, \mu}^{\prime}\left(s_{1}^{\prime}\right) \Omega_{1}^{a}\left(s_{1}^{\prime}\right) \\
& -2 g \partial^{v} \Phi_{2, \nu \mu}^{a}(z) \\
& -g \int_{0}^{s_{2}} \mathrm{~d} s_{2}^{\prime} \delta^{(4)}\left(z-y_{2}+u_{2}\left(s_{2}^{\prime}\right)\right) u_{2, \mu}^{\prime}\left(s_{2}^{\prime}\right) \Omega_{2}^{a}\left(s_{2}^{\prime}\right)
\end{aligned}
$$

and generalized to a higher number of points of Green's functions in a formally similar way. Restoring the fermionic determinant (i.e., relaxing the quenching approximation), the needed functional operation is now

$$
\begin{aligned}
& \exp \left[-\frac{i}{2} \int \frac{\delta}{\delta A} \cdot D_{\mathrm{F}}^{(0)} \cdot \frac{\delta}{\delta A}\right] \\
& \cdot \exp \left[\frac{i}{2} \int A \cdot \bar{K} \cdot A+i \int \overline{\mathrm{Q}} \cdot A\right] \cdot \exp (L[A])
\end{aligned}
$$

followed by the prescription of cancelling the potentials $A_{\mu}^{a}$, where

$$
\begin{aligned}
& \left\langle z\left|\bar{K}_{\mu \nu}^{a b}\right| z^{\prime}\right\rangle \\
& =\left[K_{\operatorname{spin}_{\mu \nu}^{a b}}^{a b}(z)+g f^{a b c} \chi_{\mu \nu}^{c}(z)\right] \delta^{(4)}\left(z-z^{\prime}\right) \\
& \quad+\left\langle z\left|\left(D_{F}^{(0)}\right)^{-1}\right|_{\mu \nu}^{a b} \mid z^{\prime}\right\rangle, \\
& \bar{Q}_{\mu}^{a}(z)=\partial^{\nu} \chi_{\nu \mu}^{a}(z)+Q_{\mu}^{a}(z) .
\end{aligned}
$$

Another differential functional identity is relevant to this operation [11]:

$$
e^{\mathfrak{D}_{A}} \mathscr{F}_{1}[A] \mathscr{F}_{2}[A]=\left(e^{\mathfrak{D}_{A}} \mathscr{F}_{1}[A]\right) e^{\overleftrightarrow{\mathfrak{D}}}\left(e^{\left.\mathfrak{D}_{A^{\prime}} \mathscr{F}_{2}\left[A^{\prime}\right]\right)}\right.
$$

where the "cross-linkage" operator $\exp \{\overleftrightarrow{\mathfrak{D}}\}$ is given by

$$
\overleftrightarrow{\mathfrak{D}}=-i \int \overleftarrow{\frac{\delta}{\delta A}} D_{\mathrm{F}}^{(0)} \frac{\vec{\delta}}{\delta A^{\prime}}
$$

and the limit of $A_{\mu}^{a}=A_{\mu}^{\prime a}=0$ is to be taken in the end. After cumbersome, still standard, calculations, one gets (up to an irrelevant factor of $\left.\exp \left((1 / 2) \operatorname{Tr} \ln \left(-D_{\mathrm{F}}^{(0)}\right)\right)\right)$ the functional statement of effective locality as

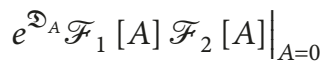

$$
\begin{aligned}
& =\mathscr{N} \exp \left[-\frac{i}{2} \int \bar{Q} \cdot \widehat{K}^{-1} \cdot \bar{Q}-\frac{1}{2} \operatorname{Tr} \ln \widehat{K}\right] \\
& \cdot \exp \left[\frac{i}{2} \int \frac{\delta}{\delta A} \cdot \widehat{K}^{-1} \cdot \frac{\delta}{\delta A}-\int \bar{Q} \cdot \widehat{K}^{-1} \cdot \frac{\delta}{\delta A}\right] \\
& \left.\cdot \exp (L[A])\right|_{A \rightarrow 0} \text {, }
\end{aligned}
$$

at $\widehat{K}_{\mu \nu}^{a b}=K_{\operatorname{spin}_{\mu \nu}}^{a b}+g f^{a b c} \chi_{\mu \nu}^{c}$, that is, locality and gauge independence again, whatever the initial gauge field function one begins with. However the famous duality relation of coupling constants $g \rightarrow 1 / g$ is not verified in QCD contrarily to the pure YM case.

This is nontrivial result, the more so if auxiliary field integrations can be carried out exactly, as in the "small case." Now these auxiliary field variables are those related to particular Fradkin's representations, and one may therefore wonder if such a property as effective locality is Fradkin's representation dependent, or not.

3.3. Effective Locality: Even More General. With the functional $L[A]$ being local and fully gauge-invariant, one may consider the case of a fermionic 2-point Green's function generalization to a higher number of points being trivial. One has thus to consider an expression like

$$
\begin{gathered}
\mathcal{N} \int_{\mathrm{d}} \mathrm{d} \int \mathrm{d}[u(s)](\cdot) \int \mathrm{d}[\chi] e^{(i / 4) \int \chi^{2}} \\
\left.e^{\mathfrak{D}_{A}^{(o)}} e^{+(i / 2) \int \chi \cdot F+(i / 2) \int A \cdot\left(D_{F}^{(0)}\right)^{-1} \cdot A} G_{\mathrm{F}}(x, y \mid A)\right|_{A \rightarrow 0},
\end{gathered}
$$

where, again, the dots in the first line stand for the integrand (26) and, this time, no particular representation is assumed for $G_{F}[A]$. Basically, this is

$$
\begin{aligned}
& \mathcal{N} \int \mathrm{d}[\chi] e^{(i / 4) \int \chi^{2}} e^{\mathfrak{D}_{A}^{(0)}} e^{+(i / 2) \int A \cdot K \cdot A+i \int A_{a}^{\mu} \partial^{\nu} \chi_{\nu \mu}^{a}} G_{\mathrm{F}}(x, y \mid \\
& A)\left.\right|_{A \rightarrow 0}, \\
& K=g f^{a b c} \chi_{\mu \nu}^{c}+\left.\left(D_{F}^{(0)}\right)^{-1}\right|_{\mu \nu} ^{a b}, \\
& \mathscr{F}_{1}[A]=\exp \left[\frac{i}{2} \int A \cdot K \cdot A+i \int A_{a}^{\mu} \partial^{\nu} \chi_{\nu \mu}^{a}\right], \\
& \mathscr{F}_{2}[A]=G_{\mathrm{F}}(x, y \mid A) .
\end{aligned}
$$

Up to a global factor of $\mathscr{N} e^{(1 / 2) \operatorname{Tr} \ln \left(-D_{\mathrm{F}}^{(0)}\right)}$ the core expression, that is, the second line of (41), is

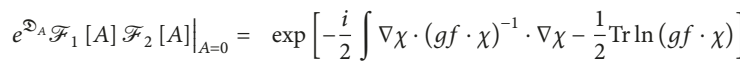

$$
\begin{aligned}
& \cdot \exp \left[\frac{i}{2} \int \frac{\delta}{\delta A} \cdot(g f \cdot \chi)^{-1} \cdot \frac{\delta}{\delta A}\right] \\
& \left.\cdot \exp \left[\int \nabla \chi \cdot(g f \cdot \chi)^{-1} \cdot \frac{\delta}{\delta A}\right] G_{\mathrm{F}}(x, y \mid A)\right|_{A \rightarrow 0} .
\end{aligned}
$$

Effective locality is manifest in the first line. In the third line, $G_{F}[A]$ is not gauge-independent but acting upon it with the two functional differentiations, followed by the prescription $A \rightarrow 0$ yielding a manifestly gauge-independent result also. As stated above, it is trivial to check that the same applies to a product of $n$ functionals, $\prod_{i=1}^{n} G_{\mathrm{F}}\left(x_{i}, y_{i} \mid A\right)$, that is, to a $2 n$-point fermionic Green's function.

One may therefore conclude that while Fradkin's representations are useful to actual calculations and derivations of further remarkable properties, the gist of effective locality does not depend on them. This independence makes of effective locality a property sound enough to support the interpretation proposed in the current article. 


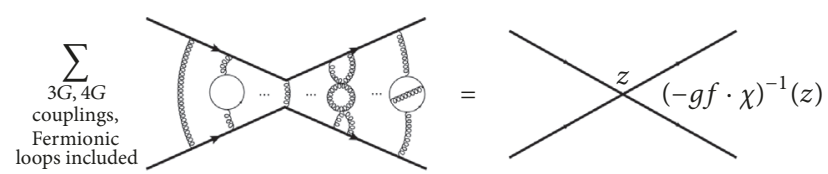

FIGURE 1: Effective Locality pictured in a rather symbolic way in which the sum of all possible QCD interactions between 2 scattering quarks "boils down" to a local contact-type interaction mediated by the structure $(-g f \cdot \chi)^{-1}(z)$.

Functional integrations (differentiations) reproduce the Feynman graphs perturbative expansions, so that, if not taken literally (analyticity in $g$ on one side, nonanalyticity on the other side), one could propose that the pictorial representation of Figure 1 means effective locality in a symbolic way.

\section{Conclusion}

Decades of experience with the abelian case of QED have probably made us forget that to calculate observables, it is intrinsically not necessary to select a gauge [15]. To maintain an explicit covariance though, for both historical and practical reasons, one has admitted to break the manifest gauge invariance in QED.

The case of QCD seems to allow one to proceed in a different way, and this is explicitly due to the nonabelian structure of QCD, with its 3 and 4 linear gluonic self-couplings. The result is a generating functional which displays covariant and gauge-independent fermionic Green's functions.

Originally derived on the basis of Fradkin's representations for the closed fermionic loops and background field fermionic propagators, the property of effective locality appears to be more general indeed. The gist of effective locality seems essentially related to the way nonabelian gauge invariance gets realized in the nonperturbative regime of QCD, and this is most easily disclosed by using functional differentiation instead of functional integration.

This situation is new and very different from the abelian case of QED or the perturbative regime of QCD: Up to complications inherent to the nonabelian structure of QCD in effect, gauge invariance follows the same indirect pattern in either cases, Slavnov-Taylor identities playing the role of Ward identities. Reaching the nonperturbative regime of QCD (intense $A_{\mu}^{a}$-fields and/or strong coupling $g \gg 1$ ) out of the perturbative sector is now recognized as a dead end because of the Gribov's copy issue which opposes a violent obstruction to this attempt $[16,17]$.

Such a difficulty does not show up in the effective locality approach, and the fact that gauge invariance in this case is direct may have something to do with the fact that the only QCD states that are outright physical are those of the nonperturbative regime of QCD, that is, states of the hadronic spectrum.

\section{Conflicts of Interest}

The authors declare that there are no conflicts of interest regarding the publication of this paper.

\section{References}

[1] H. M. Fried, Y. Gabellini, T. Grandou, and Y.-M. Sheu, "Phys. J”" C65, 395, 2010.

[2] H. M. Fried, T. Grandou, and Y.-M. Sheu, "Ann. Phys," 327, 2666, 2012.

[3] H. M. Fried, Y. Gabellini, T. Grandou, and Y.-M. Sheu, "Ann. Phys," 338, 107, 2013.

[4] H. M. Fried, T. Grandou, and Y.-M. Sheu, "Ann. Phys," 344C, 78, 2014.

[5] H. M. Fried, P. H. Tsang, Y. Gabellini, T. Grandou, and Y.-M. Sheu, "Ann. Phys," 359, 1, 2015.

[6] H. Reinhardt, K. Langfeld, and L. V. Smekal, "Phys. Lett," 300, 11, 1993.

[7] H. Reinhardt, "Dual description of QCD," in Proceedings of the conference Quark Confinement and the Hadron Spectrum II, Villa Olmo, Como, Italy, June 26-29, 1996.

[8] M. Schaden, H. Reinhardt, P. A. Amundsen, and M. J. Lavelle, "Nucl. Phys," 339, 595, 1990.

[9] C. Itzykson and J. B. Zuber, Quantum Field Theory, McGrawHill Inc., NY, USA, 1980.

[10] M. B. Halpern, “Phys. Rev," 16, 1798, 1977.

[11] H. M. Fried, Functional Methods and Models in Quantum Field Theory, the MIT Press, Cambridge, MA, USA, 1972.

[12] A. Zee, Quantum Field Theory in a Nutshell, Princeton University Press, 2nd edition, 2010.

[13] T. Grandou, “EPL," Article ID 1650120, 107, 11001, 2014.

[14] H. M. Fried, T. Grandou, and R. Hofmann, "Int. J. of Mod. Phys," 31, Nos. 20 \& 21, 1650120, 25 pages, 2016.

[15] A. Maas, "On gauge fixing," in Proceedings of the XXVIII International Symposium on Lattice Field Theory, A. Maas, Ed., Villasimius, Italy, June 2010.

[16] “XIIth Quark Confinement and the Hadron Spectrum," Thessaloniki, Greece, 28 Auust-02 September 2016.

[17] D. Zwanziger, private communication, 1st Winter Workshop in Non-Perturbative Quantum Field Theories, INLN, Valbonne, France, 18-20 January 2010, https://inis.iaea.org/search/search .aspx?orig_q=RN:45045764. 

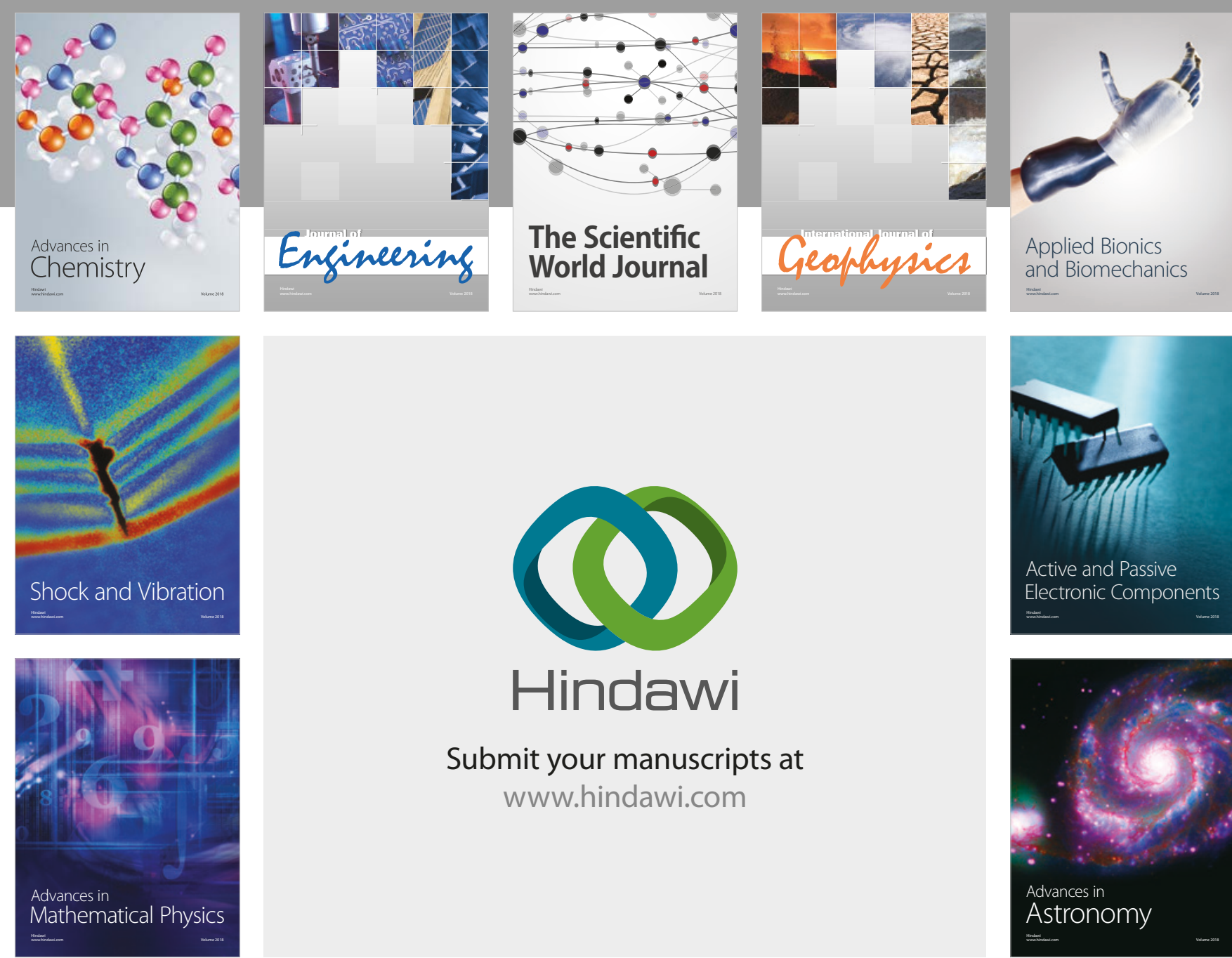

Submit your manuscripts at

www.hindawi.com

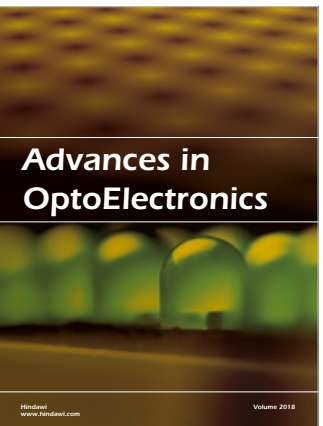

\section{Rotcting Machinery}
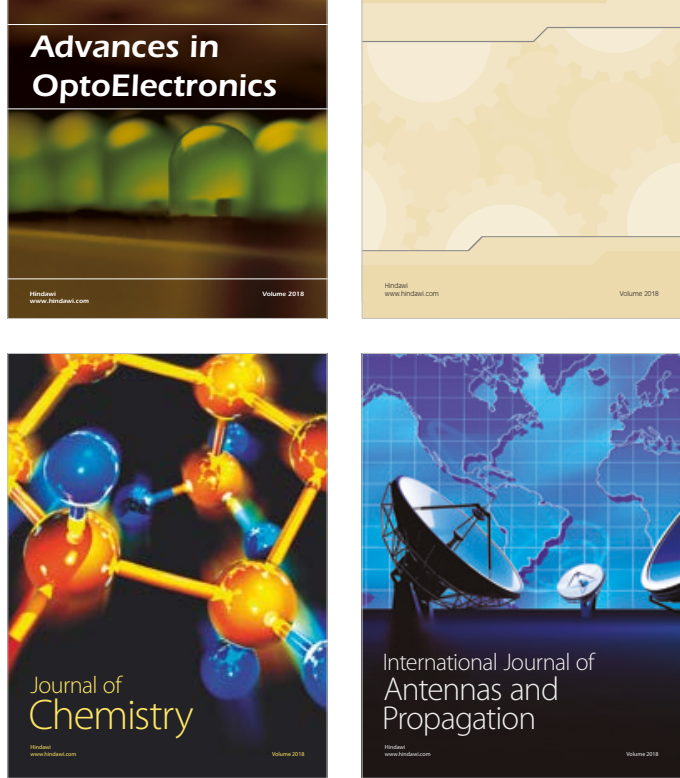

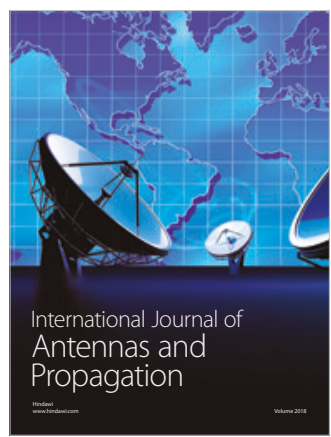

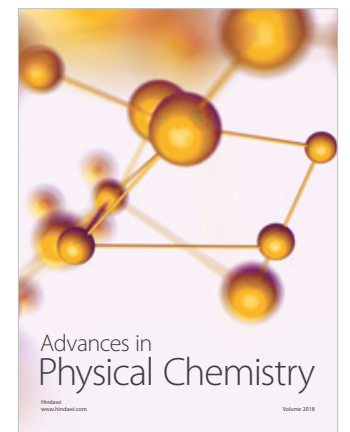

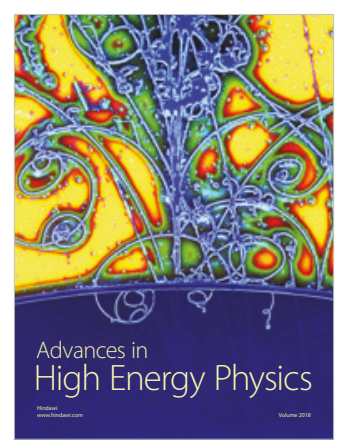

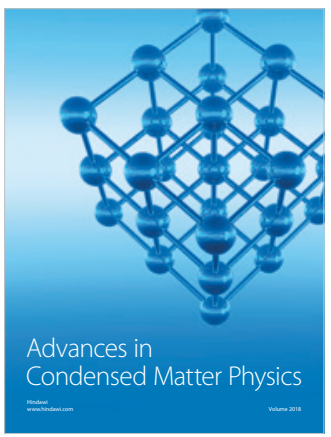

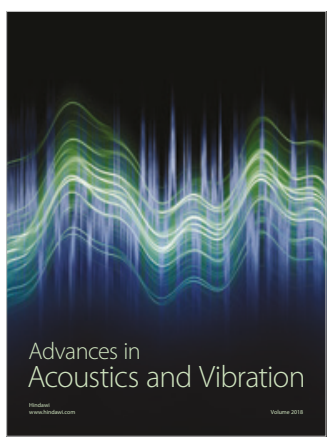

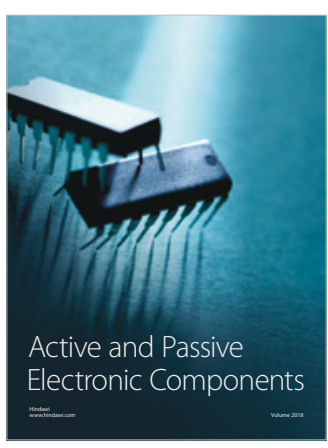
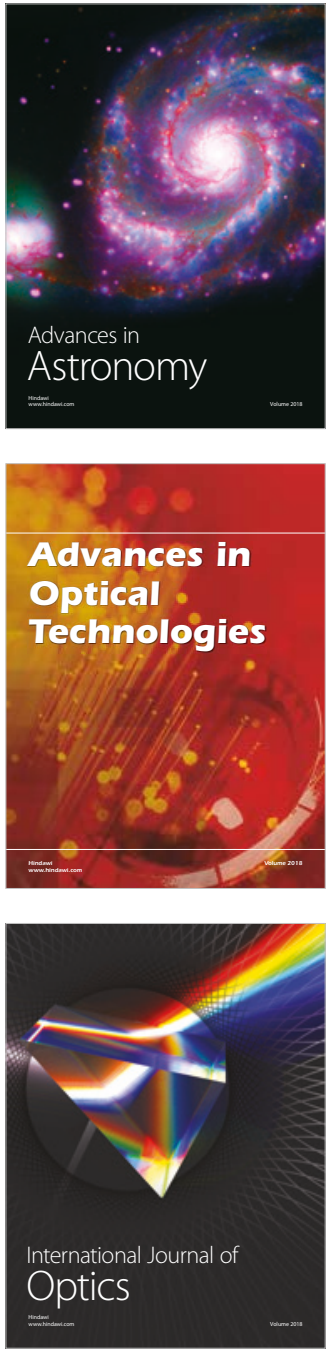